\title{
Stability of Simultaneously Block Triangularizable Switched Systems with Partial State Reset
}

\author{
Isabel Brás ${ }^{\mathrm{a} *}$, Ana C. Carapito ${ }^{\mathrm{b}}$ and Paula Rocha ${ }^{\mathrm{c}}$ \\ ${ }^{a}$ CIDMA - Center for Research and Development in Mathematics and Applications, Department of Mathematics, \\ University of Aveiro, 3810-193 Aveiro, Portugal; ${ }^{b}$ Department of Mathematics, University of Beira Interior, Portugal; \\ ${ }^{c}$ SYSTEC-Research Center for Systems \& Technologies, Faculty of Engineering, University of Porto
}

\begin{abstract}
We study the stability of a certain class of switched systems where discontinuous jumps (resets) on some of the state components are allowed, at the switching instants. It is known that, if all components of the state are available for reset, the system can be stabilizable by an adequate choice of resets. However, this question may have negative answer if there are forbidden state components for reset. We give a sufficient condition for the stabilizability of a switched system, under arbitrary switching, by partial state reset in terms of a block simultaneous triangularizability condition. Based on this sufficient condition, we show that the particular class of systems with partially commuting stable system matrices is stabilizable by partial state reset. We also provide an algorithm that allows testing whether a switched system belongs to this particular class of systems.
\end{abstract}

Keywords: switched system; stability; partial reset; simultaneous block triangularization

\section{Introduction}

Switched systems may arise either as a direct result of the mathematical modeling of a phenomenon or as the result of controller design techniques that use switching schemes, see for instance, Hespanha and Morse (2002), Hespanha, Santesso, and Stewart (2007) and Liberzon and Morse (1999). Such schemes rely on a bank of controllers supervised by a switching signal that indicates which controller should be operational at each time instant. Switching among the controllers in the bank results in a switching closed-loop system, and stability is not guaranteed even when each controller can stabilize the plant on its own.

In this paper we consider switched linear systems, these systems are defined by a family of linear time invariant (LTI) state-space systems (the bank of the switched system) together with a switching law, that determines which linear system within the family is active at each time instant.

When dealing with switched systems, the most common approach is to assume that, at each switching instant, the state evolves continuously, see, for instance, Liberzon and Morse (1999), Liberzon (2003). However, in some cases it is natural and profitable to allow discontinuous state jumps (state resets) at the switching instants. Note that state resets, also known as impulses, are present not only in switched systems, but also in many other dynamical systems where the process may experience abrupt state changes, as for instance in the case of some drug administration procedures, Lakshmikantham, Bainov, and Simeonov (1989). In the majority of these situations, the reset is either determined by the trajectory of the system, Beker, Hollot, Chait, and Han (2004), or it is time dependent, i.e., it occurs at some pre-specified time instants (regardless of the values of the state trajectory), Hetel, Daafouz, Tarbouriech, and Prieur (2013). In the case of switched systems the state reset is usually associated with the switching signal. More precisely, the reset of the state takes place at each switching instant, and is determined by which systems are

\footnotetext{
*Corresponding author. Email: ibras@ua.pt
} 
active before and after the commutation, Hespanha and Morse (2002), Hespanha et al. (2007), Yuan and Wu (2015b). In Hespanha and Morse (2002), the authors propose to make a reset in the state of a multicontroller in order to stabilize a given plant, this corresponds to considering that at each switching instant only some components of the state of the closed-loop system are available for reset, as the state of the process under control remains unchanged. Similar controller architecture may be found in Yuan and Wu (2015a) and Yuan and $\mathrm{Wu}(2015 b)$.

A switched system for which only some of the state components may jump during switching is called a switched system with partial state reset, Brás, Carapito, and Rocha (2013).

One major issue when dealing with switched systems(with or without reset) is stability. Indeed this property may not hold for the overall switched system even when each individual LTI system in the bank is stable. A stability analysis of switched systems with reset may be carried out from two standpoints. One is to consider pre-specified (partial) reset and to study the system stability, the other is to investigate how to stabilize the system, when possible, by finding a suitable (partial) reset (stabilization by reset). In this paper the second standpoint is adopted: we consider that the switching signal is arbitrary and study the stabilization by partial reset, in line with what is done in Brás et al. (2013). Note that, in many other approaches, see for instance Zhao, Yin, Li, and Niu (2015); Zhao, Zhang, Shi, and Liu (2012), where the switching is not arbitrary, the stabilization is made by picking suitable switching signals.

When dealing with the stability of a switched system (without reset), under arbitrary switching, it is frequent to use the common Lyapunov quadratic function approach. In fact, if, at each switching instant, the state evolves continuously, the existence of a common quadratic Lyapunov function (CQLF) is sufficient to ensure stability of the switched system. Several survey papers on this matter are available, see for example Liberzon and Morse (1999), Lin and Antsaklis (2009), R. Shorten, Wirth, Mason, Wulff, and King (2007). The books Liberzon (2003) and Sun (2006) are also good references on the subject.

In Brás et al. (2013), the case was considered where the switching bank consists of stable LTI statespace systems with block triangular system matrices. In that paper, it was proved that in this case a system is stabilizable by partial reset if some relevant diagonal blocks of the system matrices have CQLF; moreover a choice of suitable stabilizing resets was provided.

In this paper we are interested in investigating how to ensure stability by means of partial reset for switched systems associated to banks with simultaneously block triangularizable stable matrices. Our idea is to take advantage of the results from Brás et al. (2013). One might think that this latter problem can be easily reduced to the former one, as it would be enough to find, if possible, a simultaneously block triangularizing similarity transformation for all the system matrices in the bank; but it turns out that some similarity transformations may destroy the property of stabilization by partial reset. However, here we show that if the system bank is simultaneously block triangularizable by a similarity transformation with a $(1,1)$ invertible block, and the correspondent $(1,1)$ blocks in the bank system matrices have CQLF, then the system is stabilizable by a partial reset. Additionally, a way to construct suitable resets in order to ensure stability is given. Based on this result we identify a class of switched systems, that we call partially commuting systems, which are stabilizable by a partial reset of a certain order (the order of the reset is the number of components of the state that are allowed to jump). Note that, as shall become clear, the class of partially commuting systems is wider than the one of systems with simultaneously triangularizable matrices.

The paper is organized has follows. Preliminaries are provided in Section 2. The definition of a set of simultaneously $z$-block triangularizable matrices and a sufficient condition for the stabilization by partial reset of a switched system associated to a simultaneously block triangularizable bank are given in Section 3. In the Section 4, based on the characterization of partially commuting matrices, a class of simultaneously $z$-block triangularizable systems it is identified for which stability property is guaranteed by partial reset. Finally, a numerical example is given followed by an algorithm that determines the smallest order of a stabilizing partial reset that can be constructed based on the simultaneous block triangularization of a given set of partially commuting matrices. Conclusions are given in Section 5. In Appendix A, a detailed exposition about partially commuting matrices is given in order to clarify some of the algebraic contents of the paper. 


\section{Preliminaries}

In this section we formalize the definition of switched system (with or without reset), introduce the stability property under consideration and summarize some relevant results on stability and stabilization by reset.

Let $\mathcal{P}$ a finite index set, $\Sigma_{\mathcal{P}}=\left\{\Sigma_{p}: p \in \mathcal{P}\right\}$ a family of LTI state-space systems. Since we focus on the study of internal stability, we consider systems without inputs and disregard the output equation. Let $\dot{x}(t)=A_{p} x(t)$, where $A_{p} \in \mathbb{R}^{n \times n}$, be the state-space representation of $\Sigma_{p}$, for $p \in \mathcal{P}$. Additionally, define a switching law or a switching signal as a piecewise constant function of time $\sigma:\left[t_{0},+\infty[\rightarrow \mathcal{P}\right.$, such that, $\sigma(t)=i_{k-1}$, for $t \in\left[t_{k-1}, t_{k}\left[_{k \in \mathbb{N}}\right.\right.$; the time instants $t_{k}, k \in \mathbb{N}$, such that $t_{0}<t_{1}<\ldots<t_{k-1}<t_{k} \ldots$ are called switching instants. The set of all switching signals is represented by $\mathcal{S}_{\mathcal{P}}$. A triple $\mathbb{S}=\left(\mathcal{P}, \Sigma_{\mathcal{P}}, \mathcal{S}_{\mathcal{P}}\right)$ is said to be a switched system with switching bank $\Sigma_{\mathcal{P}}$, in the sense that each switching signal $\sigma(\cdot) \in \mathcal{S}_{\mathcal{P}}$ produces the linear time varying system $\Sigma_{\sigma}$ defined by

$$
\dot{x}(t)=A_{\sigma(t)} x(t)
$$

for all $t \geq t_{0}$, where $x(t) \in \mathbb{R}^{n}$ is the state.

This definition implies that, as is commonly assumed in most of the literature, there are no state jumps at the switching instants. Next, we present the definition of switched system with state reset, that allows a state jump at each of the switching instants; this is uniquely determined by the systems $\Sigma_{p}$ that are active before and after the switching. The state discontinuities are associated with a family of resets

$$
\mathcal{R}=\left\{\left(q, p, R_{(q, p)}\right):(q, p) \in \mathcal{P} \times \mathcal{P}, q \neq p\right\},
$$

where $R_{(q, p)}$ are non-zero real matrices of order $n$ that act on the state of the system at the switching instants. These matrices are called reset matrices.

Definition 1: A quadruple $\mathbb{S}_{R}=\left(\mathcal{P}, \Sigma_{\mathcal{P}}, \mathcal{S}_{\mathcal{P}}, \mathcal{R}\right)$ is said to be a switched system with state reset if each switching signal $\sigma(\cdot) \in \mathcal{S}_{\mathcal{P}}$ produces a linear time varying system defined as in (1), such that at each switching time instant $t_{k}$, for all $k \in \mathbb{N}$, with $t_{0}<t_{1}<\ldots<t_{k-1}<t_{k}<\ldots$,

$$
x\left(t_{k}\right)=R_{\left(i_{k-1}, i_{k}\right)} x\left(t_{k}^{-}\right), \text {if } \sigma(t)=i_{k-1} \text { for } t \in\left[t_{k-1}, t_{k}[,\right.
$$

where $x\left(t_{k}^{-}\right):=\lim _{t \rightarrow t_{k}^{-}} x(t)$ and $R_{\left(i_{k-1}, i_{k}\right)}$ are reset matrices taken according with the family of resets $\mathcal{R}$.

Clearly, according to Definition 1 , if, for all $(q, p) \in \mathcal{P} \times \mathcal{P}, R_{(q, p)}=I_{n}$, where $I_{n}$ denotes the identity matrix of order $n$, the correspondent switched system is a system without state reset, i.e., a classical switched system where the state evolves continuously. Note that for these systems (without reset) we use the notation $\mathbb{S}=\left(\mathcal{P}, \Sigma_{\mathcal{P}}, \mathcal{S}_{\mathcal{P}}\right)$, as already mentioned.

Next we present the definition of stability of a switched system (with or without state reset), under arbitrary switching. This notion is the usual global uniform exponential stability, (Liberzon, 2003, p. 22), which for linear systems with arbitrary switching is equivalent to the property of global uniform asymptotic stability, see (Liberzon, 2003, p. 22). In this paper for simplicity reasons, we use the term "stable" to refer to both equivalent properties.

Definition 2: A switched system defined by (1) and (2), is stable if there exist $\gamma, \lambda \in \mathbb{R}^{+}$such that, for every switching signal $\sigma$, for every $t_{0} \in \mathbb{R}$ and every $x_{0} \in \mathbb{R}^{n}$, the correspondent state trajectory $x(t)$, with $x\left(t_{0}\right)=x_{0}$, satisfies $\|x(t)\| \leq \gamma e^{-\lambda\left(t-t_{0}\right)}\left\|x_{0}\right\|$, for $t \geq t_{0}$.

For a switched system to be stable it is necessary to have a bank of stable invariant systems. So, in the following we exclusively consider switched systems that have a switching bank $\Sigma_{\mathcal{P}}$ formed by stable systems. 
On the other hand, a well known condition that guarantees the stability of a switched system $\mathbb{S}$ is based on the notion of common quadratic Lyapunov function. A function $V(x)=x^{T} P x$, where $P$ is a square symmetric positive definite matrix, is said to be a common quadratic Lyapunov function (CQLF) for a switched system $\mathbb{S}$ if $A_{p}^{T} P+P A_{p}<0$, for all $p \in \mathcal{P}$. With some abuse of language, we shall call the matrix $P$ a CQLF for $\Sigma_{p}$ and $A_{p}, p \in \mathcal{P}$. In fact, if at each switching instant, the state evolves continuously, the existence of a common Lyapunov quadratic function is sufficient to ensure stability of the switched system, under arbitrary switching, Khalil (1992), Liberzon and Morse (1999).

Theorem 1: If there exists a CQLF for the switched system $\mathbb{S}$, then $\mathbb{S}$ is stable.

Among the results related with the existence of a CQLF for a switched system, see for instance Liberzon, Hespanha, and Morse (1999), Narendra and Balakrishnan (1994), R. N. Shorten and Narendra (1998) and R. N. Shorten and Narendra (2002), one of the most general sufficient conditions that can be stated in simple matrix terms is the following.

Theorem 2: Let $\mathbb{S}=\left(\mathcal{P}, \Sigma_{\mathcal{P}}, \mathcal{S}_{\mathcal{P}}\right)$ be a switched system for which the switching bank $\Sigma_{\mathcal{P}}$ is associated to a set of stable simultaneously triangularizable matrices. Then there exists a CQLF for the switched system $\mathbb{S}$.

Recalling that commutative matrices are simultaneously triangularizable, Motzkin and Taussky (1952), the next result is a trivial consequence of Theorem 2, Narendra and Balakrishnan (1994), which will be relevant in the sequel.

Corollary 1: A set $\left\{A_{p}: p \in \mathcal{P}\right\}$ of stable and pairwise commuting matrices has a CQLF.

In an attempt to relax the conditions of Theorem 2, some sufficient conditions were established in terms of block matrices where the structure is block triangular or simultaneously block triangularizable, see for instance Brás, Carapito, and Rocha (2006) and Cheng, Guo, and Huang (2003). The following results are closely related to the approach used in the current paper.

Theorem 3: Let $\left\{A_{p}: p \in \mathcal{P}\right\}$ be a set of stable matrices with block triangular structure, where all diagonal blocks $A_{i i}^{p}, i=1, \ldots, t$, are square of the same order, for all $p \in \mathcal{P}$. If there exists a CQLF for each set of blocks $\left\{A_{i i}^{p}: p \in \mathcal{P}\right\}$, for $i=1, \ldots, t$, then all matrices $A_{p}$ share a CQLF.

Notice that, it is easy to conclude that the condition of previous theorem becomes a necessary and sufficient condition for the existence of CQLF with block diagonal structure.

Corollary 2: Let $\left\{A_{p}: p \in \mathcal{P}\right\}$ be a set of stable matrices. If the matrices $A_{p}$ are simultaneously block triangularizable, by a complex similarity transformation $T$, in such way that $\left\{T^{-1} A_{p} T: p \in \mathcal{P}\right\}$ satisfies the conditions of Theorem 3 then, all matrices $A_{p}$ share a CQLF.

Another result related to the existence of CQLF is the following, see Brás et al. (2013) for a proof.

Lemma 1: Let $\left\{A_{p}: p \in \mathcal{P}\right\} \subset \mathbb{R}^{n \times n}$ be a set of stable matrices and $P \in \mathbb{R}^{n \times n}$ a symmetric and positive definite matrix. Then there exists a set of invertible real matrices $\left\{S_{p}: p \in \mathcal{P}\right\}$ such that $\bar{A}_{p}=S_{p}^{-1} A_{p} S_{p}$ share $P$ as a CQLF.

Using the previous lemma, it is possible to establish the following result that ensures the stability of any switched system provided that a proper choice of resets is made and if all components of the state can be freely reset, Brás et al. (2013).

Theorem 4: Let $\mathbb{S}=\left(\mathcal{P}, \Sigma_{\mathcal{P}}, \mathcal{S}_{\mathcal{P}}\right)$ be a switched system and $S_{p}$ be invertible real matrices such that $S_{p}^{-1} A_{p} S_{p}$ share a CQLF. If $\mathcal{R}=\left\{\left(q, p, S_{p} S_{q}^{-1}\right):(q, p) \in \mathcal{P} \times \mathcal{P}, q \neq p\right\}$ then, $\mathbb{S}_{R}=\left(\mathcal{P}, \Sigma_{\mathcal{P}}, \mathcal{S}_{\mathcal{P}}, \mathcal{R}\right)$ is a stable system. 
This theorem shows that a switched system, with a bank formed by stable systems, is always stabilizable using invertible reset matrices, if all state variables are allowed to jump. However, as already mentioned, in some situations the reset of some of those components may be forbidden or inconvenient. This leads to the analysis of switched systems with state reset with a certain block structure, that is, switched systems where the reset matrices in $\mathcal{R}$ are of the form

$$
R_{(q, p)}=\left[\begin{array}{cc}
I_{n-z} & 0 \\
R_{21}^{(q, p)} & R_{22}^{(q, p)}
\end{array}\right]
$$

where the matrices $R_{22}^{(q, p)}$ are real invertible ${ }^{1}$ matrices of order $z$. In opposition to the case in which all of the state components can be subject to reset (total reset), we say that this type of reset is a partial reset of order $z$. This type of state reset appears, for instance, in Hespanha and Morse (2002) and Yuan and Wu (2015b), as a result of a multi-controller technique were the state of the controller is subject to some jump at the switching instants but the same is not allowed for the state of the process. In fact, in the aforementioned contributions, the correspondent closed-loop systems are switched systems with partial reset of the form $\left[\begin{array}{cc}I & 0 \\ 0 & \widetilde{R}_{(q, p)}\end{array}\right]$.

Contrary to what happens when all state components are free for reset, stability is not always achievable when only partial resets, as in (3), are allowed, see Example 1 in Brás et al. (2013).

Definition 3: A switched system $\mathbb{S}=\left(\mathcal{P}, \Sigma_{\mathcal{P}}, \mathcal{S}_{\mathcal{P}}\right)$ is said to be stabilizable by state reset if there exists a family of resets $\mathcal{R}$ such that the switched system with state reset $\mathbb{S}_{R}=\left(\mathcal{P}, \Sigma_{\mathcal{P}}, \mathcal{S}_{\mathcal{P}}, \mathcal{R}\right)$ is stable. In particular, if each reset matrix of $\mathcal{R}$ is an invertible matrix of the form

$$
\left[\begin{array}{cc}
I_{n-z} & 0 \\
R_{21}^{(q, p)} & R_{22}^{(q, p)}
\end{array}\right],(q, p) \in \mathcal{P} \times \mathcal{P}, z=1,2, \ldots, n
$$

the system $\mathbb{S}$ is called stabilizable by partial state reset of order $z$. When $z=n$ the partial reset becomes a total one.

As we shall see in the next section, the stabilization by partial resets is not preserved under arbitrary similar transformations, contrary to what happens in the case of total reset. However, some classes of similarity transformations preserve that property. In the following, we explore this fact in order to give some sufficient conditions for stabilization by partial reset.

\section{Stabilization by Partial Resetting and Simultaneously $z$-Block Triangularizable Switched Systems}

The following theorem gives a sufficient condition for the stabilization by partial resets for switched systems with block triangular structure; it is an immediate consequence of Corollary 1 and Corollary 2 from Brás et al. (2013).

Theorem 5: Let $\mathbb{S}=\left(\mathcal{P}, \Sigma_{\mathcal{P}}, \mathcal{S}_{\mathcal{P}}\right)$ be a block triangular switched system associated to a set of stable matrices $A_{p}=\left[\begin{array}{cc}A_{p_{11}} & A_{p_{12}} \\ 0 & A_{p_{22}}\end{array}\right]$, with $A_{p_{11}} \in \mathbb{R}^{(n-z) \times(n-z)}, p \in \mathcal{P}$. If $\left\{A_{p_{11}}: p \in \mathcal{P}\right\}$ has a CQLF, then $\mathbb{S}$ is stabilizable by partial reset with order $z$.

From this result a natural question arises: Does the conclusion of Theorem 5 still hold if the matrices $A_{p}$ are not block triangular but rather simultaneously block triangularizable? This is not a trivial question

\footnotetext{
${ }^{1}$ The situation where $R_{22}^{(q, p)}$ are not invertible raises interesting questions that will be investigated in due time.
} 
since, as the next example shows, a similarity transformation does not necessarily preserve the property of stabilization by partial reset.

Example 1: The switched system with switching bank constituted by the matrices $A_{p}=\left[\begin{array}{cc}-I_{2} & 0 \\ 0 & A_{p_{22}}\end{array}\right]$, for $p=1,2$, with $A_{1_{22}}=\left[\begin{array}{cc}-0.05 & 2 \\ -1 & -0.05\end{array}\right]$ and $A_{2_{22}}=\left[\begin{array}{cc}-0.05 & 1 \\ -2 & -0.05\end{array}\right]$ is stabilizable by partial resets of order 2 but the switched system with switching bank associated to the matrices $T^{-1} A_{p} T$, with $T=\left[\begin{array}{cc}0 & I_{2} \\ I_{2} & 0\end{array}\right]$, is not. Indeed, we have $T^{-1} A_{p} T=\left[\begin{array}{cc}A_{p_{22}} & 0 \\ 0 & -I_{2}\end{array}\right]$ and the switched system associated to the blocks $(1,1)$ is unstable, Santos (2002). So the switched system associated to the matrices $T^{-1} A_{p} T$ is not stabilizable by partial resets of order 2 .

Consequently, if we want to successfully study the partial stabilization of a switched system by applying a similarity transformation we should identify a suitable class of transformations that preserve the partial stabilization property; moreover the transformed system should be easier to analyse, with respect to partial stabilization. In the following, we shall see that such strategy can be followed with success for a certain type of simultaneous block triangularization matrices, namely those that are real and have an invertible upper right block.

Definition 4: The matrices $A_{p} \in \mathbb{R}^{n \times n}, p \in \mathcal{P}$, are said to be simultaneously $z$-block triangularizable if there exists an invertible real matrix $T=\left[\begin{array}{ll}T_{11} & T_{12} \\ T_{21} & T_{22}\end{array}\right]$, where $T_{11} \in \mathbb{R}^{(n-z) \times(n-z)}$ is invertible matrix, such that $T^{-1} A_{p} T=\left[\begin{array}{cc}\widetilde{A}_{p_{11}} & \widetilde{A}_{p_{12}} \\ 0 & \widetilde{A}_{p_{22}}\end{array}\right]$, where $\widetilde{A}_{p_{11}} \in \mathbb{R}^{(n-z) \times(n-z)}$.

A switched system with a switching bank associated to a set of simultaneously $z$-block triangularizable matrices is said to be a simultaneously z-block triangularizable switched system. Notice that, if $T$ is the identity matrix, the matrices are block triangular.

Theorem 6: Let $\mathbb{S}=\left(\mathcal{P}, \Sigma_{\mathcal{P}}, \mathcal{S}_{\mathcal{P}}\right)$ be a switched system associated to stable matrices $A_{p}, p \in \mathcal{P}$. If $\mathbb{S}$ is simultaneously $z$-block triangularizable by the matrix $T$ and all blocks $(1,1)$ of order $n-z$ of the matrices $T^{-1} A_{p} T$ have a $C Q L F$, then $\mathbb{S}$ is stabilizable by partial reset of order $z$.

Proof. Let $T=\left[\begin{array}{ll}T_{11} & T_{12} \\ T_{21} & T_{22}\end{array}\right]$ be a real invertible matrix, where $T_{11}$ is also invertible, such that

$$
\widetilde{A}_{p}=T^{-1} A_{p} T=\left[\begin{array}{cc}
\widetilde{A}_{p_{11}} & \widetilde{A}_{p_{12}} \\
0 & \widetilde{A}_{p_{22}}
\end{array}\right]
$$

and the blocks $\widetilde{A}_{p_{11}}$ have a CQLF. By Lemma 1 , there exists a set of invertible real matrices $W_{p}, p \in \mathcal{P}$, such that $W_{p}^{-1} \widetilde{A}_{p_{22}} W_{p}$ have a CQLF.

Consider $\widetilde{S}_{p}=\left[\begin{array}{cc}I & -T_{11}^{-1} T_{12} W_{p} \\ 0 & W_{p}\end{array}\right]$, then $\widetilde{S}_{p}^{-1}=\left[\begin{array}{cc}I & T_{11}^{-1} T_{12} \\ 0 & W_{p}^{-1}\end{array}\right]$. Therefore,

$$
\begin{aligned}
\bar{A}_{p} & =\widetilde{S}_{p}^{-1} \widetilde{A}_{p} \widetilde{S}_{p} \\
& =\left[\begin{array}{cc}
\widetilde{A}_{p_{11}} & -\widetilde{A}_{p_{11}} T_{11}^{-1} T_{12} W_{p}+\widetilde{A}_{p_{12}} W_{p}+T_{11}^{-1} T_{12} \widetilde{A}_{p_{22}} W_{p} \\
0 & W_{p}^{-1} \widetilde{A}_{p_{22}} W_{p}
\end{array}\right]
\end{aligned}
$$

Since $\widetilde{A}_{p_{11}}, p \in \mathcal{P}$, have a CQLF as well as $W_{p}^{-1} \widetilde{A}_{p_{22}} W_{p}, p \in \mathcal{P}$, by Theorem $3, \bar{A}_{p}, p \in \mathcal{P}$, have also a 
CQLF. Therefore, there exists $S_{p}$ such that $S_{p}^{-1} A_{p} S_{p}$ share a CQLF; more concretely

$$
\begin{aligned}
S_{p} & =T \widetilde{S}_{p} \\
& =\left[\begin{array}{cc}
T_{11} & 0 \\
T_{12} & C W_{p}
\end{array}\right],
\end{aligned}
$$

$C=T_{22}-T_{21} T_{11}^{-1} T_{12}$ is the Schur complement of the block $T_{11}$ in $T$. Then, by Theorem 4, the system $\mathbb{S}_{R}=\left(\mathcal{P}, \Sigma_{\mathcal{P}}, \mathcal{S}_{\mathcal{P}}, \mathcal{R}\right)$ is stable, for the partial resets:

$$
\begin{aligned}
R_{(q, p)} & =S_{p} S_{q}^{-1} \\
& =\left[\begin{array}{cc}
T_{11} & 0 \\
T_{12} & C W_{p}
\end{array}\right]\left[\begin{array}{cc}
T_{11}^{-1} & 0 \\
-W_{q}^{-1} C^{-1} T_{12} T_{11}^{-1} & W_{p}^{-1} C^{-1}
\end{array}\right] \\
& =\left[\begin{array}{cc}
I & 0 \\
\left(I-C W_{p} W_{q}^{-1} C^{-1}\right) T_{12} T_{11}^{-1} & C W_{p} W_{q}^{-1} C^{-1}
\end{array}\right],
\end{aligned}
$$

so $\mathbb{S}$ is stabilizable by partial reset of order $z$.

Notice that, the previous proof is constructive. In fact, it gives a way to construct a set of invertible partial reset matrices that stabilizes the system.

\section{Partially commuting switched systems}

In this section we identify a class of systems that are simultaneously $z$-block triangularizable and for which the transformed $(1,1)$ blocks share a CQLF. Note that, in order to have a $z$-block triangularizable switched system, the following conditions should be satisfied:

(i) the matrices $A_{p}, p \in \mathcal{P}=\{1, \cdots, N\}$, must have a common invariant subspace $\mathcal{N}$ of dimension $n-z$

(ii) if $T=\left[\begin{array}{lllll}v_{1} & \ldots & v_{n-z} & \ldots & v_{n}\end{array}\right]$, where $\left\{v_{1}, \ldots, v_{n-z}\right\}$ is a basis of $\mathcal{N}$ and $\left\{v_{1}, \ldots, v_{n}\right\}$ is a basis of $\mathbb{R}^{n}$, and hence $T^{-1} A_{p} T=\left[\begin{array}{cc}\widetilde{A}_{p_{11}} & \widetilde{A}_{p_{12}} \\ 0 & \widetilde{A}_{p_{22}}\end{array}\right]$, then $T=\left[\begin{array}{cc}T_{11} & T_{12} \\ T_{21} & T_{22}\end{array}\right]$ is a real matrix with $T_{11}$ invertible.

If additionally, the $(1,1)$ blocks $\widetilde{A}_{p_{11}}$ commute pairwise, by Corollary 1 , they have a CQLF. So, using Theorem 6 , we may conclude that the system is stabilizable by partial reset of order $z$. Bearing this in mind, we are interested in characterizing the sets of matrices $\left\{A_{p}: p \in \mathcal{P}\right\}$ that share a common invariant subspace where they commute; this is equivalent to the existence of a common eigenvector. A characterization of the sets of two matrices with a common eigenvector was made in Shemesh (1984) and generalized for a finite number of matrices in Jamiołkowski and Pastuszak (2015). Matrices that have a common eigenvector are said to be partially commuting matrices, (Alpin, Elsner, \& Ikramov, 2000). See Appendix A for more details on this. Note that, simultaneous triangularizable matrices and pairwise commutative matrices are particular classes of partially commuting matrices. In the sequel, for the sake of simplicity, we consider systems where $N=2$. For our purposes the following characterization of partially commuting matrices is the most suitable one (it is an immediate consequence of Theorem 9 of Appendix A).

Theorem 7: Let $A_{1}, A_{2} \in \mathbb{R}^{n \times n}$ and ${ }^{2} L=\sum_{k, l=1}^{n-1}\left[A_{1}^{k}, A_{2}^{l}\right]^{T}\left[A_{1}^{k}, A_{2}^{l}\right]$. The matrices $A_{1}$ and $A_{2}$ are partially commuting matrices if and only if $\operatorname{rank} L<n$.

\footnotetext{
${ }^{2}[X, Y]$ stands for the traditional commutator of two square matrices $X$ and $Y$, i.e., $[X, Y]=X Y-Y X$.
} 
The following corollary gives an easy test for the stabilizability by partial reset of order $z$ of a partially commuting system for which rank $L=z$.

Corollary 3: Let $A_{1}, A_{2} \in \mathbb{R}^{n \times n}$ be partially commuting stable matrices such that $\operatorname{rank} L=z$ and let $\left\{v_{1}, \ldots, v_{n-z}\right\}$ be a basis of ker $L$. If $\left[\begin{array}{l}T_{11} \\ T_{21}\end{array}\right]=\left[v_{1} \cdots v_{n-z}\right]$, where $T_{11} \in \mathbb{R}^{(n-z) \times(n-z)}$ is invertible, then the switched system $\mathbb{S}=\left(\mathcal{P}, \Sigma_{\mathcal{P}}, \mathcal{S}_{\mathcal{P}}\right)$ associated to $A_{1}$ and $A_{2}$ is stabilizable by partial reset of order $z$.

Proof. By hypothesis, see Apeendix A (specially Theorem 11 and Corollary 4), there exists an invertible matrix $T=\left[\begin{array}{ll}T_{11} & T_{12} \\ T_{21} & T_{22}\end{array}\right]$, such that $T^{-1} A_{p} T=\left[\begin{array}{cc}\widetilde{A}_{p_{11}} & \widetilde{A}_{p_{12}} \\ 0 & \widetilde{A}_{p_{22}}\end{array}\right], p \in\{1,2\}$, where $\widetilde{A}_{p_{11}} \in \mathbb{R}^{(n-z) \times(n-z)}$ commute. Since $T_{11}$ is invertible, by Theorem 6 , then the switched system $\mathbb{S}$ is stabilizable by partial reset of order $z$.

Next we present an illustrative example that shows an application of the previous corollary, and motivates a slightly more general result as well as the algorithm that closes this section.

Example 2: Let $\mathbb{S}$ be the switched system with switching bank associated to the stable matrices:

$$
A_{1}=\left[\begin{array}{cccc}
-6.0 & 0 & 1.0 & 2.0 \\
-4.0 & -8.0 & -6.9 & 0.1 \\
4.0 & 7.9 & 4.8 & -2.1 \\
-4.0 & -3.9 & -0.9 & 2.0
\end{array}\right] \text { and } A_{2}=\left[\begin{array}{cccc}
-4.0 & -3.0 & -4.0 & -3.0 \\
-2.0 & -8.3109 & -8.5563 & -4.2454 \\
2.0 & 8.2109 & 4.4563 & 0.24539 \\
-2.0 & -6.2109 & -2.5563 & -0.34539
\end{array}\right] \text {. }
$$

In the following, the presented results were obtained using floating-point computations in MATLAB, (so, most of the equal signs must be interpreted as approximately equal). The matrix $L$ associated to the matrices $A_{1}$ and $A_{2}$, defined as $L=\sum_{k, l=1}^{3}\left[A_{1}^{k}, A_{2}^{l}\right]^{T}\left[A_{1}^{k}, A_{2}^{l}\right]$, is a real $4 \times 4$ matrix such that rank $L=3<4$ so $A_{1}$ and $A_{2}$ are partially commuting matrices. ker $L$ is generated by $v_{1}=\left[\begin{array}{llll}-0.5 & -0.5 & 0.5 & -0.5\end{array}\right]^{T}$. Since $-0.5 \neq 0$, we may conclude by the previous corollary that the system is stabilizable by partial reset of order 3. In fact, considering three more vectors, for instance a set of generators of the orthogonal subspace of $\operatorname{ker} L$,

$$
\begin{aligned}
& v_{2}=\left[\begin{array}{llll}
-0.5 & 0.83333 & 0.16667 & -0.16667
\end{array}\right]^{T} \\
& v_{3}=\left[\begin{array}{llll}
0.5 & 0.16667 & 0.83333 & 0.16667
\end{array}\right]^{T} \\
& v_{4}=\left[\begin{array}{llll}
-0.5 & -0.16667 & 0.16667 & 0.83333
\end{array}\right]^{T},
\end{aligned}
$$

the matrix $T=\left[\begin{array}{llll}v_{1} & v_{2} & v_{3} & v_{4}\end{array}\right]$ is such that

$$
T^{-1} A_{1} T=\left[\begin{array}{cc}
-5 & * \\
0 & \widetilde{B}_{1_{22}}
\end{array}\right] \text { and } T^{-1} A_{2} T=\left[\begin{array}{cc}
-6 & \widetilde{B}^{*} \\
0 & \widetilde{B}_{22}
\end{array}\right]
$$

where $*$ denotes some matrices (with no special importance to us) and

$$
\widetilde{B}_{1_{22}}=\left[\begin{array}{ccc}
-5.0333 & -4.9667 & -1.9333 \\
4.9333 & 2.8667 & -0.066667 \\
-0.93333 & 1.0333 & -0.033333
\end{array}\right] \text { and } \widetilde{B}_{2_{22}}=\left[\begin{array}{ccc}
-3.3555 & -3.6282 & -2.2727 \\
3.2555 & -0.47183 & -1.7273 \\
-1.2555 & 2.3718 & 1.6273
\end{array}\right] \text {; }
$$

Clearly, the blocks $(1,1):-5$ and -6 have a CQLF.

Now, notice that the matrices $\widetilde{B}_{1_{22}}$ and $\widetilde{B}_{2_{22}}$ may be still partially commuting matrices, see Appendix A for details on this. This can be tested using exactly the procedure used above for the matrices $A_{1}$ and $A_{2}$. 
The matrix $L$ associated to the matrices $\widetilde{B}_{1_{22}}$ and $\widetilde{B}_{2_{22}}$, defined as $L=\sum_{k, l=1}^{2}\left[\widetilde{B}_{1_{22}}^{k}, \widetilde{B}_{2_{22}}^{l}\right]^{T}\left[\widetilde{B}_{1_{22}}^{k}, \widetilde{B}_{2_{22}}^{l}\right]$, is such that $\operatorname{rank} L=2<3$ and its kernel is generated by $v_{1}=\left[\begin{array}{lll}0.57735 & -0.57735 & 0.57735\end{array}\right]^{T}$. Similarly, taking $V=\left[\begin{array}{ccc}0.57735 & 0.57735 & -0.57735 \\ -0.57735 & 0.78868 & 0.21132 \\ 0.57735 & 0.21132 & 0.78868\end{array}\right]$, we update the matrix $T$ as

$$
T:=T\left[\begin{array}{cc}
1 & 0 \\
0 & V
\end{array}\right]=\left[\begin{array}{cccc}
-0.5 & -0.8660 & 0.0000 & 0.0000 \\
-0.5 & 0.2887 & 0.5774 & -0.5774 \\
0.5 & -0.2887 & 0.7887 & 0.2113 \\
-0.5 & 0.2887 & 0.2113 & 0.7887
\end{array}\right] \text {. }
$$

Then, by computing $T^{-1} A_{1} T$ and $T^{-1} A_{2} T$, we arrive to a $2 \times 2$ upper block triangular structure:

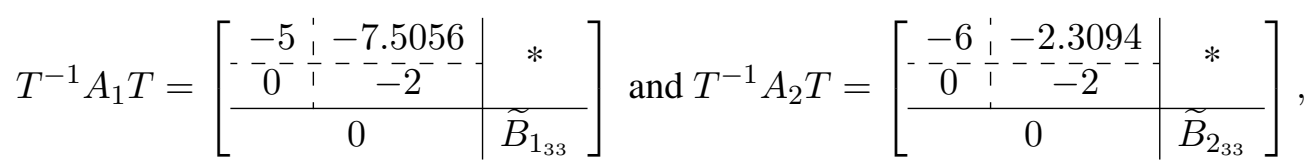

where

$$
\widetilde{B}_{1_{33}}=\left[\begin{array}{cc}
-0.1 & -1.464 \\
5.464 & -0.1
\end{array}\right] \text { and } \widetilde{B}_{2_{33}}=\left[\begin{array}{cc}
-1.832 & -2.464 \\
4.464 & 1.632
\end{array}\right]
$$

and the block $(1,1)$ of order 2 in $T, T_{11}=\left[\begin{array}{cc}-0.5 & -0.8660 \\ -0.5 & 0.2887\end{array}\right]$ has clearly rank $2(=3-1)$. Furthermore, by construction (and as it can be easily seen) the $2 \times 2$ matrices in the left upper corners have CQLF. In this case we have obtained two triangular matrices. At this point we may conclude that the system is stabilizable by partial reset of order 2 and check if the remaining blocks $\widetilde{B}_{1_{33}}$ and $\widetilde{B}_{2_{33}}$ are still partially commuting matrices. The correspondent matrix $L=\left[\widetilde{B}_{1_{33}}, \widetilde{B}_{2_{33}}\right]^{T}\left[\widetilde{B}_{1_{33}}, \widetilde{B}_{2_{33}}\right]$ is such that $\operatorname{rank} L=2$. So, these blocks are no longer partially commuting. Using this iterative procedure we concluded that the system is stabilizable by partial reset of order 2 .

As we have illustrated in the previous example, if two real matrices $A_{1}$ and $A_{2}$ are partially commuting matrices, they can be brought by a simultaneous similarity transformation, $\mathrm{T}$, into block upper triangular matrices where a certain number of corresponding diagonal blocks commute, i.e,

$$
T^{-1} A_{1} T=\left[\begin{array}{cc}
\widetilde{A}_{1_{11}} & \widetilde{A}_{1_{12}} \\
0 & \widetilde{A}_{1_{22}}
\end{array}\right] \text { and } T^{-1} A_{2} T=\left[\begin{array}{cc}
\widetilde{A}_{2_{11}} & \widetilde{A}_{2_{12}} \\
0 & \widetilde{A}_{2_{22}}
\end{array}\right], \text { respectively, }
$$

where $\widetilde{A}_{1_{11}}$ and $\widetilde{A}_{2_{11}}$ are block triangular matrices where the corresponding diagonal blocks have the same order and commute, (see Appendix A for details).

Definition 5: Let $\mathbb{S}=\left(\mathcal{P}, \Sigma_{\mathcal{P}}, \mathcal{S}_{\mathcal{P}}\right)$ be a switched system with switching bank associated to partially commuting stable matrices $A_{1}$ and $A_{2}$. $\mathbb{S}$ is called a $(n-z)$-partially commuting system if there exists a real similarity transformation $T=\left[\begin{array}{ll}T_{11} & T_{12} \\ T_{21} & T_{22}\end{array}\right]$, with $T_{11} \in \mathbb{R}^{(n-z) \times(n-z)}$ invertible, such that

$$
T^{-1} A_{p} T=\left[\begin{array}{cc}
\widetilde{A}_{p_{11}} & \widetilde{A}_{p_{12}} \\
0 & \widetilde{A}_{p_{22}}
\end{array}\right], p=1,2
$$

where $\widetilde{A}_{1_{11}}$ and $\widetilde{A}_{2_{11}}$ are block triangular matrices, with the same block triangular structure, having com- 
mutative correspondent diagonal blocks.

Using this definition, by Corollary 1, Theorem 3 and Theorem 6, the following can be stated.

Theorem 8: Let $\mathbb{S}=\left(\mathcal{P}, \Sigma_{\mathcal{P}}, \mathcal{S}_{\mathcal{P}}\right)$ be a switched system associated to stable matrices $A_{p}, p=1,2$. If $\mathbb{S}=\left(\mathcal{P}, \Sigma_{\mathcal{P}}, \mathcal{S}_{\mathcal{P}}\right)$ is a $(n-z)$-partially commuting switched system then $\mathbb{S}$ is stabilizable by partial state reset of order $z$.

Based on the previous considerations, we present a constructive algorithm that tests if a given switched system is $(n-z)$-partially commuting for some $z$, which implies the possibility of stabilization by a partial reset of order $z$. More precisely, this algorithm returns the smallest order of a stabilizing partial reset that can be constructed based on simultaneous block triangularization with partially commuting diagonal blocks. The process is descending, until possible, and will have as many iterations as the number of blocks appearing in the canonical forms presented in Theorem 12 of Appendix A. In the algorithm, the matrices $G_{1}$ and $G_{2}$ are playing the same role as the blocks $\widetilde{B}_{1_{i i}}$ and $\widetilde{B}_{2_{i i}}, i=2,3$, in Example 2, and the matrices $A_{1}, A_{2}$ are stable.

\section{Algorithm}

Initiation step: Set $T:=I_{n}, G_{1}:=A_{1}, G_{2}:=A_{2}, n:=\operatorname{size}\left(A_{1}\right), g:=n$ and $h:=n$.

Step 1: Compute $L=\sum_{k, l=1}^{n-1}\left[G_{1}^{k}, G_{2}^{l}\right]^{T}\left[G_{1}^{k}, G_{2}^{l}\right]$ and set $r:=\operatorname{rank} L$.

If $r=0$, write "the system is stable" and stop.

If $r=g$, write "the system is stabilizable by partial reset of order $h$ " and stop.

Else, go to Step 2.

Step 2: Compute a basis for $\operatorname{ker} L$ and denote it by $\left\{v_{1}, \ldots, v_{g-r}\right\}$.

Step 3: Choose $v_{g-r+1}, \ldots, v_{g}$ such that $V:=\left[\begin{array}{llllll}v_{1} & \ldots & v_{g-r} & v_{g-r+1} & \ldots & v_{g}\end{array}\right]$ is invertible. Set $V:=$ $\left[\begin{array}{cc}I_{n-g} & 0 \\ 0 & V\end{array}\right]$

Step 4: Set $T:=T V, g:=r, T_{11}:=\left[\begin{array}{ll}I_{n-g} & 0_{(n-g) \times g}\end{array}\right] T\left[\begin{array}{c}I_{n-g} \\ 0_{g \times(n-g)}\end{array}\right]$;

If $\operatorname{rank} T_{11}=n-g$, set $h:=g$.

Else, go to Step 5.

Step 5: Set $G_{j}:=\left[\begin{array}{ll}0 & I_{g}\end{array}\right] T^{-1} A_{j} T\left[\begin{array}{c}0 \\ I_{g}\end{array}\right], j=1,2$. Return to Step 1.

Remark 1: Note that this algorithm may fail to deliver the conclusion that the system is stabilizable by partial reset of order $z$ even if the conditions of Theorem 6 are satisfied, as there exist simultaneously $z$ block triangularizable systems for which the blocks $(1,1)$ of $T^{-1} A_{p} T$ have a CQLF, but are not partially commuting systems. In fact, $(n-z)$-partially commuting systems with stable bank are a particular class of systems that satisfy the conditions of Theorem 6 . On the other hand, in some cases, this procedure makes possible to conclude about the stability of the switched system, namely when it is easy to see that the final matrices $G_{1}$ and $G_{2}$ have a CQLF, (cf Theorem 3). This is, for instance, the case when $G_{1}$ and $G_{2}$ are $2 \times 2$, and $G_{1} G_{2}$ and $G_{1} G_{2}^{-1}$ have no real negative eigenvalues, R. N. Shorten and Narendra (2002), and the case when $\operatorname{rank}\left(G_{1}-G_{2}\right)=1$ and $G_{1} G_{2}$ have no real negative eigenvalues King and Nathanson (2006). Note that, using this criteria, in Example 2, we may conclude the matrices $A_{1}$ and $A_{2}$ do not have CQLF, since the final two diagonal blocks do not have CQLF. 


\section{Conclusion}

In this paper, we have considered switched systems that allow jumps in some state components when switching occurs. In particular, we have analysed the case where these jumps are dictated by a certain linear rule called a partial state reset. The central question was to verify if a switched system is stabilizable using partial reset of certain order. This question was examined for block simultaneously triangularizable switched systems. In particular, the stabilization of partially commuting systems was analysed. An algorithm was proposed that determines the smallest order of a stabilizing partial reset that can be constructed based on simultaneous block triangularization with partially commuting diagonal blocks. This criterion is somehow a generalization of the sufficient condition presented in Narendra and Balakrishnan (1994), for stability of switched systems involving commutative matrices. We note that the obtained conditions based on partial commutation or on simultaneous block triangularization are still restrictive. Nevertheless, we suspect that obtaining general conditions, within an algebraic approach (like the one used here) will prove to be difficult.

\section{References}

Alpin, Y., Elsner, L., \& Ikramov, K. (2000). On condensed forms for partially commuting matrices. Linear Algebra Appl., 306(1-3), 165-182.

Beker, O., Hollot, C., Chait, Y., \& Han, H. (2004). Fundamental properties of reset control systems. Automatica, 40(6), $905-915$.

Brás, I., Carapito, A. C., \& Rocha, P. (2006). Stability of interconnected switched systems. In Proceedings of the 7th portuguese conference on automatic control (pp. 1-6).

Brás, I., Carapito, A. C., \& Rocha, P. (2013). Stability of switched systems with partial state reset. IEEE Trans. Automat. Contr., 58(4), 1008-1012.

Cheng, D., Guo, L., \& Huang, J. (2003). On quadratic Lyapunov functions. IEEE Transactions on Automatic Control, 48, 885-890.

Hespanha, J. P., \& Morse, A. S. (2002). Switching between stabilizing controllers. Automatica, 38, 1905-1917.

Hespanha, J. P., Santesso, P., \& Stewart, G. (2007). Optimal controller initialization for switching between stabilizing controllers. In Proc. of the 46th ieee conference on decision and control (p. 5511-5516).

Hetel, L., Daafouz, J., Tarbouriech, S., \& Prieur, C. (2013). Stabilization of linear impulsive systems through a nearly-periodic reset. Nonlinear Analysis: Hybrid Systems, 7(1), 4 - 15.

Jamiołkowski, A., \& Pastuszak, G. (2015). Generalized Shemesh criterion, common invariant subspaces and irreducible completely positive superoperators. Linear and Multilinear Algebra, 63(2), 314-325.

Khalil, H. (1992). Non linear systems. Macmillan Co., New York.

King, C., \& Nathanson, M. (2006). On the existence of a common quadratic Lyapunov function for a rank one difference. Linear Algebra and its Applications, 419, 400-416.

Lakshmikantham, V., Bainov, D., \& Simeonov, P. S. (1989). Theory of impulsive differential equations. World Sientific. 
Liberzon, D. (2003). Switching in systems and control. Birkhäuser Boston.

Liberzon, D., Hespanha, J. P., \& Morse, A. S. (1999). Stability of switched systems: a Lie-algebraic condition. Systems Control Letters, 37(3), 117-122.

Liberzon, D., \& Morse, A. S. (1999). Basic problems in stability and design of switched systems. IEEE Control Systems, 19, 59-70.

Lin, H., \& Antsaklis, P. J. (2009). Stability and stabilizability of switched linear systems: a survey of recent results. IEEE Transactions on Automatic Control, 54, 308-322.

Motzkin, T. S., \& Taussky, O. (1952). Pairs of matrices with property L. Trans. Amer. Math. Soc., 73, $108-114$.

Narendra, K. S., \& Balakrishnan, J. (1994). A common Lyapunov function for stable LTI systems with commuting $\mathcal{A}$-matrices. IEEE Transactions on Automatic Control, 39(4), 2469-.2471.

Santos, E. (2002). Estabilidade em esquemas de controlo comutado. Unpublished master's thesis, Universidade de Aveiro.

Shemesh, D. (1984). Common eigenvectors of two matrices. Linear Algebra Appl., 62, 11-18.

Shorten, R., Wirth, F., Mason, O., Wulff, K., \& King, C. (2007). Stability criteria for switched and hybrid systems. SIAM review, 49(4), 545-592.

Shorten, R. N., \& Narendra, K. S. (1998). On the stability and existence of common Lyapunov functions for stable linear switching systems. In 37th ieee conference on decision and control (pp. 3723-3724).

Shorten, R. N., \& Narendra, K. S. (2002). Necessary and sufficient condictions for the existence of a commom quadratic Lyapunov function for a finite number of stable second order linear time-invariant systems. Int. J. Adapt. Control Signal Process, 16, 709-728.

Sun, Z. (2006). Switched linear systems: Control and design. Springer.

Yuan, C., \& Wu, F. (2015a). Asynchronous switching output feedback control of discretetime switched linear systems. International Journal of Control, 1-9. Retrieved from http://dx.doi.org/10.1080/00207179.2015.1016454 (Published online: 16 Mar 2015)

Yuan, C., \& Wu, F. (2015b). Hybrid control for switched linear systems with average dwell time. Automatic Control, IEEE Transactions on, 60(1), 240-245.

Zhao, X., Yin, S., Li, H., \& Niu, B. (2015, Jan). Switching stabilization for a class of slowly switched systems. Automatic Control, IEEE Transactions on, 60(1), 221-226. doi:

Zhao, X., Zhang, L., Shi, P., \& Liu, M. (2012, July). Stability and stabilization of switched linear systems with mode-dependent average dwell time. Automatic Control, IEEE Transactions on, 57(7), 1809-1815. doi:

\section{Appendix A. Maximal invariant subspace where a finite set of matrices commute and partially commuting matrices}

Let $\mathcal{A}=\left\{A_{p}: p \in \mathcal{P}\right\}$ be a set of real matrices of order $n$ and $\mathcal{M}$ be a subspace of $\mathbb{R}^{n \times n}$. $\mathcal{M}$ is said to be $\mathcal{A}$ - invariant if it is $A_{p}$-invariant for ever $p \in \mathcal{P}$. The matrices $A_{p}, p \in \mathcal{P}$ are said to commute (pairwise) on $\mathcal{M}$ if $\left(A_{i} A_{j}-A_{j} A_{i}\right) x$, for all $x \in \mathcal{M}$. Note that there exists always an invariant subspace where a set of matrices commute, that subspace is the zero space. The existence and characterization of non trivial subspaces with such properties were first studied in Shemesh (1984), for sets of two complex matrices. In fact, in that paper Shemesh was mainly concerned with an equivalent problem: the existence of a common eigenvector of two complex matrices. The characterization of the sets of two matrices with a common eigenvector is the following, where $[X, Y]$ is the standard notation for the commutator $X Y-Y X$ of the matrices $X$ and $Y$ :

Theorem 9 (Shemesh (1984)): The matrices $A_{1}, A_{2} \in \mathbb{C}^{n \times n}$ have a common eigenvector if and only if

$$
\mathcal{N}=\bigcap_{i, j=1}^{n-1} \operatorname{ker}\left[A_{1}^{i}, A_{2}^{j}\right] \neq\{0\} .
$$

Recently, the following generalization of Shemesh's theorem for a higher number of matrices has been given, its proof essentially mimics the Shemesh's proof. 
Theorem 10 ( Jamiołkowski and Pastuszak (2015)): Let $A_{1}, A_{2}, \ldots, A_{N}$ be a set matrices in $\mathbb{C}^{n \times n}$. The matrices $A_{1}, A_{2}, \ldots, A_{N}$ have a common eigenvector if and only if

$$
\mathcal{N}=\bigcap_{\substack{k_{1}+k_{2}+\ldots+k_{s} \neq 0 \\ l_{1}+l_{2}+\ldots+l_{s} \neq 0 \\ k_{j}, l_{j} \geq 0}}^{n-1} \operatorname{ker}\left[A_{1}^{k_{1}} \ldots A_{s}^{k_{s}}, A_{1}^{l_{1}} \ldots A_{s}^{l_{s}}\right] \neq\{0\}
$$

For our purposes, the properties of the nonzero subspace $\mathcal{N}$ are more important that the theorems themselves. Furthermore, since our matrices are real and we want to work with subspaces in $\mathbb{R}^{n}$, we must carefully analyze the arguments used in order to see if they still hold. For sake of simplicity, we consider the case $N=2$. The next result reflects that analysis; the proof that we present is slightly different from the one presented in Shemesh (1984).

Theorem 11: Let $A_{1}, A_{2}$ be matrices in $\mathbb{R}^{n \times n}$ such that $\mathcal{N}=\bigcap_{i, j=1}^{n-1} \operatorname{ker}\left[A_{1}^{i}, A_{2}^{j}\right]$ is a nonzero subspace of $\mathbb{R}^{n}$. Then $\mathcal{N}$ is an $\left\{A_{1}, A_{2}\right\}$-invariant subspace where $A_{1}$ and $A_{2}$ commute. Moreover, $\mathcal{N}$ is the maximal subspace with that property.

Proof. First note that, using Caley-Hamilton Theorem, $\mathcal{N}=\bigcap_{i, j \in \mathbb{N}} \operatorname{ker}\left[A_{1}^{i}, A_{2}^{j}\right]$. If $x \in \mathcal{N}$,

$$
\begin{aligned}
{\left[A_{1}^{i}, A_{2}^{j}\right] A_{1} x } & =A_{1}^{i} A_{2}^{j} A_{1} x-A_{2}^{j} A_{1}^{i} A_{1} x \\
& =A_{1}^{i} A_{1} A_{2}^{j} x-A_{2}^{j} A_{1}^{i+1} x \\
& =0, \text { for every } i, j \in \mathbb{N} .
\end{aligned}
$$

So, $\mathcal{N}$ is $A_{1}$-invariant. Similarly, it can be shown that $\mathcal{N}$ is $A_{2}$-invariant. Therefore, $\mathcal{N}$ is $\left\{A_{1}, A_{2}\right\}$ invariant. The commutativity of the matrices on $\mathcal{N}$ is trivial, since if $x \in \mathcal{N}, x \in \operatorname{ker}\left[A_{1}, A_{2}\right]$.

It remains to prove that $\mathcal{N}$ is the maximal invariant subspace where $A_{1}$ and $A_{2}$ commute. Let $W$ be a subspace of $\mathbb{R}^{n}$ such that $W$ is $\left\{A_{1}, A_{2}\right\}$-invariant and $A_{1} A_{2} x=A_{2} A_{1} x$, for every $x \in W$. Next we prove that $W \subset \mathcal{N}$ by proving that $W \subset \operatorname{ker}\left[A_{1}^{i}, A_{2}^{j}\right]$, for every $i, j \in \mathbb{N}$. First, let us prove that, by induction,

$$
W \subset \operatorname{ker}\left[A_{1}^{i}, A_{2}\right], i \in \mathbb{N} \text {. }
$$

In fact, for $i=1$ (A1) holds. Suppose that $W \subset \operatorname{ker}\left[A_{1}^{k}, A_{2}\right]$, i.e., for all $x \in W, A_{1}^{k} A_{2} x=A_{2} A_{1}^{k} x$. Then, for all $x \in W$,

$$
\begin{aligned}
A_{1}^{k+1} A_{2} x-A_{2} A_{1}^{k+1} x & =A_{1}^{k+1} A_{2} x-A_{2} A_{1}^{k} \underbrace{A_{1} x}_{\in W} \\
& =A_{1}^{k+1} A_{2} x-A_{1}^{k} A_{2} A_{1} x \\
& =A_{1}^{k+1} A_{2} x-A_{1}^{k+1} A_{2} x \\
& =0
\end{aligned}
$$

So $W \subset \operatorname{ker}\left[A_{1}^{k+1}, A_{2}\right]$. Therefore, by induction, that $W \subset \operatorname{ker}\left[A_{1}^{i}, A_{2}\right], i \in \mathbb{N}$. Now, let us consider a fixed $i \in \mathbb{N}$, and prove, by induction (considering that (A1) holds), that

$$
W \subset \operatorname{ker}\left[A_{1}^{i}, A_{2}^{j}\right], j \in \mathbb{N} .
$$


In fact, for $j=1$ (A2) holds, because (A1) holds. Suppose that $W \subset \operatorname{ker}\left[A_{1}^{i}, A_{2}^{k}\right]$, i.e., for all $x \in W$, $A_{1}^{i} A_{2}^{k} x=A_{2}^{k} A_{1}^{i} x$. Then, for all $x \in W$,

$$
\begin{aligned}
A_{1}^{i} A_{2}^{k+1} x-A_{2}^{k+1} A_{1}^{i} x & =A_{1}^{i} A_{2}^{k} \underbrace{A_{2} x}_{\in W}-A_{2}^{k+1} A_{1}^{i} x \\
& =A_{2}^{k} A_{1}^{i} A_{2} x-A_{2}^{k+1} A_{1}^{i} x \\
& =A_{2}^{k+1} A_{1}^{i} x-A_{2}^{k+1} A_{1}^{i} x \\
& =0
\end{aligned}
$$

So $W \subset \operatorname{ker}\left[A_{1}^{i}, A_{2}^{k+1}\right]$. Therefore, by induction, it follows that $W \subset \operatorname{ker}\left[A_{1}^{i}, A_{2}^{j}\right], i, j \in \mathbb{N}$.

Notice that the subspace $\mathcal{N}$ may be written as $\mathcal{N}=\operatorname{ker} L$, where

$$
L=\sum_{i, j=1}^{n-1}\left[A_{1}^{i}, A_{2}^{j}\right]^{T}\left[A_{1}^{i}, A_{2}^{j}\right]
$$

and $\operatorname{dim} \mathcal{N}=n-r$, with $r=\operatorname{rank} L$. Consequently, the next corollary follows,

Corollary 4: Let $\mathcal{A}=\left\{A_{1}, A_{2}\right\}$ be a set matrices in $\mathbb{R}^{n \times n}$. If $\operatorname{rank} L=r$, for $r \neq n$, then there exists an invertible matrix $T \in \mathbb{R}^{n \times n}$ such that

$$
\begin{gathered}
T^{-1} A_{1} T=\left[\begin{array}{cc}
B_{1_{11}} & \widetilde{B}_{1_{12}} \\
0 & \widetilde{B}_{1_{22}}
\end{array}\right] \\
T^{-1} A_{2} T=\left[\begin{array}{cc}
B_{2_{11}} & \widetilde{B}_{2_{12}} \\
0 & \widetilde{B}_{2_{22}}
\end{array}\right],
\end{gathered}
$$

where the blocks $B_{1_{11}}, B_{2_{11}} \in \mathbb{R}^{(n-r) \times(n-r)}$ commute.

This way of obtaining block triangular matrices by a simultaneous similarity transformation, where the blocks $(1,1)$ commute, is described in Alpin et al. (2000). In this paper, matrices that have a common eigenvector (which is equivalent to $\mathcal{N} \neq\{0\}$ ) are called partially commuting matrices. Notice that, if $r=0$ the initial matrices are already commutative, and in this degenerated case the blocks $(2,2)$ must be considered missing and $T$ may be considered to be the identity matrix. Otherwise, the blocks $\widetilde{B}_{1_{22}}$ and $\widetilde{B}_{2_{22}}$ may be commutative or not. If they are not commutative, they might be partially commutative matrices. In case $\widetilde{B}_{1_{22}}$ and $\widetilde{B}_{2_{22}}$ are partially commutative matrices, the same type of simultaneous block triangularization is possible. Therefore, in that case, there exists an invertible matrix $S$ such that

$$
\begin{array}{r}
S^{-1} A_{1} S=\left[\begin{array}{ccc}
B_{1_{11}} & B_{1_{12}} & \widetilde{B}_{1_{13}} \\
0 & B_{1_{22}} & \widetilde{B}_{1_{23}} \\
0 & 0 & \widetilde{B}_{1_{33}}
\end{array}\right] \\
S^{-1} A_{2} S=\left[\begin{array}{ccc}
B_{2_{11}} & B_{2_{12}} & \widetilde{B}_{2_{13}} \\
0 & B_{2_{22}} & \widetilde{B}_{2_{23}} \\
0 & 0 & \widetilde{B}_{2_{33}}
\end{array}\right],
\end{array}
$$

where the blocks $B_{1_{i i}}, B_{2_{i i}}, i=1,2$, commute. This process may be repeated (depending on partial commutativity of the blocks $(3,3)$ ) and so on until possible. Eventually, with this procedure, $A_{1}$ and $A_{2}$ will be brought by a simultaneous similarity transformations into block upper triangular matrices where a certain 
number of corresponding diagonal blocks commute. In order to be more precise, we state the following theorem, which is the version for real matrices of the one stated for complex matrices in (Alpin et al., 2000).

Theorem 12: Let $A_{1}, A_{2} \in \mathbb{R}^{n \times n}$ be partially commutative matrices. Then, for some positive integer $k$, there exists an invertible matrix $T \in \mathbb{R}^{n \times n}$ such that

$$
\begin{aligned}
T^{-1} A_{1} T & =\left[\begin{array}{cccc}
B_{1_{11}} & B_{1_{12}} & \ldots & B_{1_{1 k}} \\
0 & B_{1_{22}} & \ldots & B_{1_{2 k}} \\
\vdots & \vdots & \ddots & \vdots \\
0 & 0 & \ldots & B_{1_{k k}}
\end{array}\right] \\
T^{-1} A_{2} T & =\left[\begin{array}{cccc}
B_{2_{11}} & B_{2_{12}} & \ldots & B_{2_{1 k}} \\
0 & B_{2_{22}} & \ldots & B_{2_{2 k}} \\
\vdots & \vdots & \ddots & \vdots \\
0 & 0 & \cdots & B_{2_{k k}}
\end{array}\right],
\end{aligned}
$$

where the blocks $B_{1_{i i}}, B_{2_{i i}}, i=1, \ldots, k-1$, commute. The last blocks $B_{1_{k k}}$ and $B_{2_{k k}}$ either commute or do not partially commute. The number of blocks and its sizes are uniquely determine by $A_{1}$ and $A_{2}$. 